\title{
Evaluación de Sobrecarga Postural en Trabajadores: Revisión de la Literatura
}

\author{
WORKERS POSTURAL OVERLOAD ASSESSMENT: LITERATURE REVIEW
}

Bettina Patricia López Torres', Elvia Luz González Muñoz², Cecilia Colunga Rodríguez³, Eduardo Oliva López

1. Instituto Mexicano del Seguro Social. Doctorado en Ciencias de Salud en el Trabajo, Universidad de Guadalajara, Guadalajara, Jalisco, México.

2. Centro Universitario de Arte, Arquitectura y Diseño, Universidad de Guadalajara. México.

3. Hospital de Pediatría Centro Médico Nacional del Occidente, Instituto Mexicano del Seguro Social. Instituto de Investigación en Salud Ocupacional,

Centro Universitario de Ciencias de la Salud, Universidad de Guadalajara. México.

4. SEPI-ESIME, Instituto Politécnico Nacional, Ciudad de México D.F.

\section{RESUMEN}

Introducción. Las lesiones músculo-esqueléticas tienen un enorme y creciente impacto en el mundo. Son la mayor causa de dolor y discapacidad. Debido a su alta prevalencia y a su asociación con otras morbilidades, causan un importante impacto socioeconómico. Objetivo. Describir el estado que guarda la investigación nacional e internacional acerca de sobrecarga postural en trabajadores, mediante la identificación y análisis de publicaciones científicas especializadas. Método. Revisión documental descriptiva analítica. Se incluyeron documentos del año 1999 al 2012, publicados en bases de datos y revistas electrónicas en idioma inglés, español y portugués, utilizando las palabras clave sobrecarga postural, evaluación ergonómica y métodos ergonómicos. Se seleccionaron 50 artículos. Resultados. Las publicaciones fueron 5 en portugués, 20 en inglés y 25 en español. Los métodos ya existentes son de utilidad para identificar sobrecarga postural, en diversas actividades económicas. Conclusiones. No existe hasta el momento un método de evaluación integral, cuya aplicación sea sencilla, y los resultados más completos, que incluyan más variables para evaluar la sobrecarga postural. Esto sugiere la necesidad de proponer nuevos métodos.

(López B, González E, Colunga C, López E, 2014. Evaluación de Sobrecarga Postural en Trabajadores: Revisión de la Literatura. Cienc Trab. MayAgo; 16 [50]: 111-115).

Palabras claves: SOBRECARGA POSTURAL, EVALUACIÓN ERGONÓMICA, MÉTODOS ERGONÓMICOS.

\section{ABSTRACT}

Introduction. Musculoskeletal injuries have a huge and growing impact on the world. They are the main cause of pain and disability. Due to its high prevalence and its association with other morbidities, they cause an important economic impact. Objective. Describing the state of the national and international research on workers postural overload through the identification and analyzing specialized scientific publications. Method. Review of descriptive analytical documents. Documents included were from 1999 to 2012, published in electronic databases and journals in English, Spanish and Portuguese languages, using the keywords postural overload and ergonomic assessment and ergonomic methods. 50 articles were selected. Results. 5 Publications were in Portuguese, 20 in English and 25 in Spanish. Existing methods are useful for identifying postural overload in various economic activities. Conclusions. There is no a method of comprehensive evaluation so far, which has a simple implementation and better results, including more variables to assess postural overload. This suggests the need for proposing new methods.

Key words: POSTURAL OVERLOAD, ERGONOMIC ASSESSMENTS, ERGONOMIC METHODS.

\section{INTRODUCCIÓN}

Las lesiones músculo-esqueléticas tienen un enorme y creciente impacto a nivel mundial, desde la perspectiva de productividad y economía de la industria. Son la principal causa de dolor y discapacidad; debido a su alta prevalencia y a su asociación con

Correspondencia / Correspondence:

Bettina Patricia López Torres

Calle 13 Num. 11 Col. Porvenir Delegación Azcapotzalco,

Código Postal 02940,

Ciudad de México, Distrito Federal, México.

e-mail: bettapatt@yahoo.com.mx

Tel.: (01) 0445513010586

Recibido: 29 de mayo de 2014 / Aceptado: 28 de Junio 2014 otras morbilidades, ocasionan un importante impacto socioeconómico. ${ }^{1}$ Según datos reportados por Kumar ${ }^{2}$, cerca del 58\% de la población mundial mayor a los 10 años de edad pasa un tercio de su tiempo en el trabajo, generando 21,6 trillones de dólares como producto interno bruto que sostiene la economía mundial; asimismo, se estima que del 30 al 50\% de los trabajadores está expuesto a riesgos ocupacionales que le puede generar lesiones músculo-esqueléticas. ${ }^{2}$

En México, la patología músculo-esquelética es de las primeras causas de morbilidad, tal como lo establece la evidencia de acuerdo a las estadísticas del IMSS del año 2011, en donde se reporta que el número de riesgos de trabajo en total fue de 536,322 casos. $^{3}$

La sobrecarga postural en el trabajador se caracteriza porque este se encuentra fuera de la posición corporal neutra por un determinado tiempo, lo que favorece la presencia de sintomatología de dolor, inflamación, disestesias, parestesias y limitación del 
trabajador para realizar su trabajo, llegando a impedir la realización de actividades cotidianas, obligando al trabajador a solicitar incapacidad temporal para el trabajo, lo que genera ausentismo, disminución en la productividad, pérdidas económicas y, principalmente, daños a la salud de forma importante. La lumbalgia es un problema frecuentemente encontrado en salas de urgencias en nivel de atención médica primaria. Es la causa más común de limitación de actividad en individuos menores de 45 años de edad. Se estima que de la población total, entre el $60 \%$ y el $80 \%$ sufrirá por lo menos un episodio de dolor agudo de espalda. ${ }^{1}$ No obstante la frecuencia de problemas músculo-esqueléticos en el ámbito laboral, se sabe que la evaluación ergonómica se realiza mediante los métodos existentes más representativos, tales como Ovako Working Analysis System (OWAS), Rapid Entire Body Assessment (REBA), Mutua de la Agrupación de Propietarios de Fincas Rústicas de España (MAPFRE), Modelo Simple Integral (MODSI), Rapid Upper Limb Assessment (RULA), entre otros.

El objetivo de la presente investigación fue describir el estado que guarda la investigación nacional e internacional acerca de sobrecarga postural en trabajadores, mediante la identificación y análisis de publicaciones cientificas especializadas, las cuales basan su aporte en la aplicación de los métodos de evaluación ergonómica existentes y el diseño de propuestas para los mismos fines.

\section{MATERIAL Y MÉTODOS}

Se realizó un estudio de tipo documental, descriptivo y analítico, mediante la búsqueda de publicaciones electrónicas en revistas, libros, tesis y difusión en congresos y presentaciones inéditas, en idioma inglés, español y portugués, entre los años 1999 y 2012, a través de bases de referencia tales como Google académico, PubMed, así como Revista electrónica Scielo, Revista electrónica Elsevier, Revista electrónica Applied Ergonomics, Revista electrónica Ciencia \& Trabajo, y el Instituto Nacional de Seguridad e Higiene en el Trabajo de España, utilizando las palabras claves sobrecarga postural, evaluación ergonómica y métodos ergonómicos.

Para seleccionar las publicaciones científicas a analizar, se aplicaron los siguientes criterios:

- Inclusión: a) Publicaciones relacionadas con posturas forzadas, sobrecarga postural, carga física, análisis ergonómico, evaluación ergonómica y aplicación de métodos de evaluación ergonómica tales como OWAS, REBA, MAPFRE, MODSI, RULA, etc. b) Publicaciones en artículos de revistas, libros, tesis, ponencias o presentaciones inéditas. c) Textos completos. d) Que se encontraran de forma electrónica sin costo. e) En idioma inglés, español y portugués. f) Fecha de publicación comprendida entre el año 1999 y el 2012.

- Exclusión: a) Costo para accesar. b) Publicaciones en idioma diferente al inglés, español y portugués.

Se obtuvieron 50 publicaciones que cumplieron los criterios de inclusión y exclusión; en relación al tiempo de publicación se consideraron publicaciones desde el año 1999 hasta el 2012, esto para estar en posibilidad de incluir a los autores pioneros en el tema de investigación. Se contabilizó una publicación de cada uno de los siguientes países: Irán, Italia, Chile, Turquía y Perú; dos publicaciones realizadas en Taiwán, Colombia, Cuba, Suecia,
Reino Unido y Países Bajos; tres publicaciones de Estados Unidos de América y de la India; cuatro publicaciones de México; siete publicaciones de Brasil y España; y nueve publicaciones de Venezuela, encontrándose 5 publicaciones en idioma portugués, veinte en inglés y veinticinco en español. Se identificaron 11 publicaciones en donde se aplicó el método OWAS, 3 con el método REBA, 3 con el método MODSI, 1 con MAPFRE, 15 de validación de instrumentos para evaluación ergonómica de sobrecarga postural, 4 con aplicación de otros métodos de evaluación ergonómica, y 13 en donde se aplican más de dos métodos de evaluación ergonómica.

Cabe mencionar que las referencias fueron redactadas de acuerdo al estilo de la American Psychological Association (APA), derivado de que es una forma estandarizada de organizar la información, desde su obtención hasta su registro en el documento generado por la investigación. ${ }^{4}$

\section{RESULTADOS}

Del total de las publicaciones analizadas, 11 de ellas se basaron en la aplicación del método OWAS, método utilizado en diversas poblaciones de trabajadores, tales como las relacionadas a la industria de la construcción, manejo de buques, empleados administrativos, de limpieza, estibadores y desestibadores, construcción civil, carpinteros, confección, mantenimiento y conductores de autobuses; evaluando desde un caso en una publicación, hasta 722 grupos de buques, y desde 119 hasta 2880 posturas. Concluyendo que, según hallazgos publicados, este método es una herramienta ergonómica de mucha utilidad, ya que permitió identificar posturas forzadas que representan sobrecarga postural. ${ }^{5-15}$

De acuerdo a lo reportado por las 3 investigaciones que aplicaron el método REBA esto se realizó en poblaciones de 30 hasta 55 trabajadores en diversos giros, tales como industria petrolera lacustre, aserradero y trabajo con videoterminales, se concluye que el método REBA es una herramienta ergonómica de utilidad para la identificación de sobrecarga postural, en diversas actividades económicas, hasta en trabajadores administrativos que hacen uso de equipo de cómputo. ${ }^{16-18}$

En lo referente a la aplicación del método MODSI, se identificaron 3 investigaciones, las cuales fueron realizadas en diversas actividades dentro de la industria automotriz, tales como servicios, aluminio, ensambladores de autopartes y reciclaje, oscilando la evaluación entre 31 y 37 puestos de trabajo y observando hasta 54 trabajadores. ${ }^{19-21}$

Respecto al método MAPFRE, sólo se obtuvo un artículo publicado, en el cual se evaluó a población de trabajadores que intervienen en un proceso quirúrgico, tanto área médica como área de enfermería. La evaluación se hizo con una duración de dos horas y treinta minutos, concluyendo que este método no ha sido muy aplicado para identificación de sobrecarga postural; sin embargo, los autores refieren que es de utilidad para identificar el grado de riesgo de exposición para desarrollar lesiones músculo-esqueléticas, señalando que es necesario la aplicación de otros métodos complementarios. $^{22}$

Por otra parte, en cuanto a las 4 publicaciones que se identificaron en relación a la evaluación de otros métodos para sobrecarga postural, se observó que fueron evaluados desde 15 hasta 11,054 trabajadores de un aserradero, siendo analizadas diversas 
áreas de producción, tales como aserradero, madereras, fundición y una muestra de población abierta de España. En estas investigaciones, se analizaron los estudios que no estaban considerados previamente por la metodología que aplican para la identificación de sobrecarga postural, encontrando que son de utilidad para los fines buscados. ${ }^{23-26}$

En lo relativo a las 15 publicaciones identificadas para la validación de instrumentos, se observó que se evaluaron empresas con 19 actividades, y que tienen desde 20 hasta 6000 trabajadores; adicionalmente, se identifican diversos tipos de empresa, entre las que destacan construcción, textil, trabajo de oficina, y actividad en computadora. Posterior al análisis de estos artículos, se estableció que hay diversas propuestas de herramientas de evaluación ergonómica, que si bien en su primer aplicación dejan algunas variables sin considerar, es necesario llevar a cabo observaciones posteriores en diversas poblaciones de trabajadores, con la finalidad de identificar su utilidad en la detección del grado de riesgo músculo-esquelético. Por otro lado, es de importancia mencionar que el proceso que se lleva a cabo para la validación de una herramienta de evaluación, es similar en todos los estudios analizados. ${ }^{27-41}$

Finalmente, en lo referente a los últimos 15 artículos agrupados en publicaciones que aplican varios métodos de evaluación ergonómica (dos o más métodos), se observaron desde 5 trabajadores en la pesca hasta 162 operarios de armado en la producción, en diversos giros empresariales tales como pesca, automotriz, aluminio, odontología, transportistas, estibadores, cargadores, mecánicos, operarios y ayudantes, ensamble y proveedores, mercado ambulante, fundición, producción de alimentos, armado de productos y en área de la salud. Posterior al análisis de estas investigaciones, se identifica que cuando se aplica más de un método se obtienen resultados más completos que se integran entre sí, lo que determina el grado de riesgo de lesión músculoesquelética de forma más objetiva $\mathrm{y}$, adicionalmente, se puede ver que al aplicar métodos para la identificación de otro tipo de riesgos ergonómicos, la identificación de los riesgos de exposición se amplía y permite proponer medidas de control. ${ }^{42-54}$

\section{DISCUSIÓN}

En relación a los estudios con aplicación del método OWAS, de igual manera, se observó que el método es de ayuda para identificar el grado de riesgo para desarrollar lesiones músculo-esqueléticas y mostrar, como lo refiere Saraji et $\mathrm{al}^{7}$, en estudios a empresas con diferentes actividades económicas que, para una evaluación ergonómica más completa, se requiere de la aplicación de otros métodos adicionales, y no solo de uno.
Adicionalmente, de acuerdo a lo reportado por la aplicación del método REBA, se observó que se aplica la herramienta a la par con otros métodos para obtener una evaluación integral e identificar todos los factores de riesgo a que están expuestos los trabajadores en el ejercicio de sus actividades, tal como lo menciona Troconis et al en el año 2008. ${ }^{17}$

De acuerdo a lo revisado por aplicación del método MODSI, se concluye que es una herramienta de reciente creación que, aunque se encuentra aún en proceso de validación, es de fácil aplicación, permitiendo la identificación del grado de riesgo de lesión músculo-esquelética en diversas actividades industriales, como lo explica Manero y cols. en diferentes investigaciones del año 2008 a 2011.19-21,43,47

En las investigaciones respecto al método RULA, se reporta la aplicación de métodos tales como entrevistas, cuestionarios y el propio método, los cuales muestran resultados subjetivos, en el caso de la entrevista y los cuestionarios, así como resultados objetivos, tal es el caso de RULA, como lo menciona Soares et $\mathrm{al}^{24}$, para obtener el grado de riesgo de lesiones músculo-esqueléticas.

\section{CONCLUSIONES}

Para evaluar la sobrecarga postural, existen métodos de evaluación ergonómica que fueron creados para aplicarse por personal con cierto conocimiento del área, los cuales tienen características especiales, de acuerdo a las variables a evaluar, pero que se complementan entre sí, por lo que se aplican generalmente más de dos métodos para obtener resultados completos y objetivos, razón por la cual se considera que no existe hasta el momento un método de evaluación integral, de aplicación sencilla, y con resultados más integrales, abarcando mayor número de variables para evaluar la presencia de sobrecarga postural, otorgando resultados objetivos que generaren a corto, mediano y largo plazo propuestas de mejora para mantener o, en su caso, mejorar las condiciones laborales de los trabajadores desde la perspectiva ergonómica abriendo, además, la posibilidad de nuevas líneas de investigación. Por otro lado, se observa que la validación de instrumentos para la evaluación ergonómica constituye un proceso complejo, que requiere de diversas aplicaciones, y que es una buena opción para generar una propuesta al respecto. Por lo anteriormente expuesto, se considera que se dio cumplimiento al objetivo establecido al inicio de la investigación, que fue describir el estado que guarda la investigación nacional e internacional acerca de sobrecarga postural en trabajadores, mediante la identificación y análisis de publicaciones científicas especializadas, siendo que se encuentra aplicación de los métodos en diversas poblaciones. 
1. Fabiani I. Prevalencia de Patología Músculo-Esquelética Reumatoidea en el CESFAM "Cristo Vive". [Tesis de maestria no publicada]. Santiago: Universidad de Chile; 2006.

2. Kumar S. Biomechanics in Ergonomics. United States of America: Taylor \& Francis; 2008.

3. México. Instituto Mexicano del Seguro Social. Memoria estadistica 2009. México, D.F.: IMSS; 2010

4. Zavala S. Guía a la redacción en el estilo APA, 6ta ed. San Juan: Biblioteca de la Universidad Metropolitana de Cupey; 2012.

5. Li K-W, Lee C-L. Postural analysis of four jobs on two building construction sites: an experience of using the OWAS Method in Taiwan. J. Occup Health. 1999:41:183-190.

6. Velandia E, Muñoz J. Factores de riesgo de carga física y diagnóstico de alteración osteomuscular en trabajos de minas de carbón en el valle de Ubaté. Ciencias de la Salud. 2004:2(1): 24-32.

7. Saraji J, Hassanzadeh M, Pourmahabadian M, Shahtaheri S. Evaluation of Musculoskeletal Disorders Risk Factors Among The Crew Of The Iranian Ports And Shipping Organization's Vessels. Acta Med Iran. 2004:42(5):350354.

8. Serrano W, Lázaro E, Valero $\mathrm{H}$. Trastornos musculoesqueléticos relacionados con las condiciones de trabajo de estibadores y operadores de equipos montacargas en el puerto de la Habana. Revista Cubana de Salud y Trabajo. 2005:6(1):19-26.

9. Kumar $R$, Chaikumarn $M$, Lundberg J. Participatory Ergonomics and an Evaluation of a Low-Cost Improvement Effect on Cleaners' Working Posture. Int J Occup Saf Ergo. 2005:11(2):203-210.

10. Ávila S. Evaluación ergonómica de los puestos de estibador y desestibador en una empresa embotelladora de la Ciudad de México. [Tesis no publicada]. México: UNAM- IMSS; 2005.

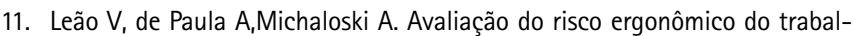
hador da construção civil durante a tarefa do levantamento de paredes. XIII SIMPEP - Bauru, SP, Brasil, 2006 [en línea]. Sao Paulo:UNESP; 2007.[Citado may 2014]. Disponible en: http://www.simpep.feb.unesp.br/anais/anais_13/ artigos/

12. Gilkey D, Keefe $T$, Bigelow $P$, Herron $R$, Duvall $K$, Hautaluoma et al. Low Back Pain Among Residential Carpenters: Ergonomic Evaluation using OWAS and 2D Compression Estimation. Int J Occup Saf Ergo. 2007:13(3):305-321.

13. De Paula A, Da Silva J, Semensato C, Carneiro L. Avaliação de Risco Ergonômico em Indústria de Confecção a través do Método de Análise Postural Ovaco Working Posture Analysing System -OWAS. 5to Congreso Internacional de Pesquisa em Design. Baurú, 10-12 outubro 2009 [en línea]. Sao Paulo: UNESP; 2010. Disponible en: http://www.faac.unesp.br/ ciped2009/anais/Ergonomia

14. Da Silva A, De Oliveira D, De Assis G, Fernandes J Resende R. Analise Ergonômica E Aplicação Do Método OWAS Em Uma Oficina De Manutenção Mecânica De Uma Usina Termoelétrica. XXX Encontro Nacional De Engenharia De Produção, Sao Paulo, 12-15 outubro, 2010 [en línea]. Sao Paulo: ABEPRO; 2011 [citado may 2014]. Disponible en: http://www.abepro.org.br/ biblioteca/enegep2010_tn_sto_116_759_16490.pdf

15. Domínguez M. Daños a la salud en los conductores de autobuses en una empresa de transporte urbano de Barcelona [Tesis no publicada][en línea]. Barcelona: Universidad Pompeu Fabra; 2010 [citada may 2014]. Disponible en: www.upf.edu/cisal/_pdf/TFM_Maritza_Dominguez

16. Pillastrini $P$, Mugnai $R$, Farneti $C$, Bertozzi $L$, Bonfiglioli $R$, Curti $S$ et al. Reducing Musculoskeletal Complaints in Operators Evaluation of Two Preventive Interventions for of Video Display Terminals. Phys Ther. 2007:87(5):535-545.

17. Troconis F, Lubo A, Montiel M, Quevedo A, Rojas L, Chacin B et al. Valoración postural y riesgo de lesión músculo-esquelética en trabajadores de una plataforma de perforación petrolera lacustre. Salud trab. 2008:16(1): 29-38.

18. Anas A, Qutubuddin S, Hebbal S, Kumar A. An ergonomic study of work related musculoskeletal disorders among the workers working in typical Indian saw mills. Int J Engine Res Develop. 2012:3(9):38-42.
19. Manero $R$, Rodríguez T. Comportamiento del Modelo Simple Integral (MODSI) en la búsqueda del nivel de riesgo de lesiones musculoesqueléticas en diferentes sectores industriales. Reporte cientifico [en línea]. Caracas: ORP; 2008[citado dic 2013]. Disponible en: http://www.prevencionintegral. com/Articulos/@Datos/_ORP2008

20. Rodríguez $E$, Manero R. Evaluación integral del nivel de riesgo músculoesquelético en diferentes actividades laborales. Salud de los trabajadores. 2008:16(1):17-28.

21. Manero $R$, Rodríguez $T$, Colotto $M$. Análisis del modelo simple e integral (MODSI) como instrumento de evaluación del riesgo a lesiones músculoesqueléticas. Trauma Fund MAPFRE. 2011: 22(1):47-53.

22. Jota D, Molina I. Propuesta De Rediseño Ergonómico Del Quirófano Tipo Abdominal Del Hospital Militar De Caracas "Dr. Carlos Arvelo". [Tesis no publicada] [en linea].Caracas: Universidad Central de Venezuela; 2003 [citado may 2014]. Disponible en: http://saber.ucv.ve/jspui/ bitstream/123456789/479/1/Tesis.pdf

23. Chamblás L, Vargas J. Estudio Ergonómico en Serruchos Trozadores y Finger-Joint en una Empresa Maderera. Cienc Trab. 2005:7(18): 40-47.

24. Soares $A$, Bakke $H$, Farias $G$. Riesgos biomecánicos en posturas de trabajadores de un aserradero. Fisioter Pesqui. 2009:16(1): 28-33.

25. Ciudad-Valls I. Prevalencia de trastornos musculoesqueléticos y factores asociados en la población ocupada en España. [Tesis no publicada][en linea]. Barcelona: Universidad de PompeuFabra- CISP; 2011 [citada may 2014]. Disponible en: http://www.upf.edu/cisal/_pdf/TFM_Ivxn_Ciudad

26. Singh J, Lal H, Kocher G. Musculoskeletal Disorder Risk Assessment in small scale forging Industry by using RULA Method. Int J Engine Adv Tech. 2012:1(5): 513-518.

27. Hignett S, McAtamney L. Rapid Entire Body Assessment (REBA). Appl Ergon. 2000:31:201-205.

28. Pinzke S. Automatic registration of OWAS postures from video film. Proceedings of the Human Factors and Ergonomics Society. Pro Quest Psychology Journals. 2000:6:529-532.

29. Lee YH, Su MC. Design and validation of a desk-free and posture-independent input device. Appl Ergon. 2004:39:399-406.

30. Simonelli $A_{1}$ Camarotto J. Método de análise de tarefasindustriais como ferramenta para a inclusão de portadores de necesidades especiais no trabalho. Rev Ter Ocup Univ São Paulo. 2005:16(3):137-146.

31. Janowitza I, Gillenb M, Ryanc G, Rempela D, Trupinc L, Swigc et al. Measuring the physical demands of work in hospital settings: Design and implementation of an ergonomics assessment. Appl Ergon. 2006:37: 641-658.

32. Vispe $C$, Bascuas J, Martínez B, Alcalde V. Propuesta de un método de valoración de daño por lesión musculoesquelética como instrumento de prevención terciaria en el medio laboral. MAPFRE MEDICINA. 2007:18(1): 42-52.

33. David G, Woods V, Li G, Buckle P. The development of the Quick Exposure Check (OEC) for assessing exposure to risk factors for work-related musculoskeletal disorders. Appl Ergon. 2008; 39:57-69.

34. Kitis A, Celik E, Aslan U,Zencir M. DASH questionnaire for the analysis of musculoskeletal symptoms in industry workers: A validity and reliability study. Appl Ergon. 2008:40: 251-255.

35. Ijmkera S, Mikkersc J, Blattera B, van der Beeka A, van Mechelena W et al. Test-retest reliability and concurrent validity of a web-based questionnaire measuring workstation and individual correlates of work postures during computer work. Appl Ergon. 2008: 39: 685-696.

36. Baker N, Cook J,Redfern M. Rater reliability and concurrent validity of the Keyboard Personal Computer Style instrument (K-PeCS). Appl Ergon. 2009:40: 136-144.

37. Vázquez L, Escárcega J, Medina A. Sistema de evaluación ergonómica, para estaciones de trabajo con ensamble manual, en los procesos de producción, en la industria maquiladora del Noreste del Estado de Sonora. XV Congreso Internacional de Ergonomía SEMAC, Ciudad Juárez abr. 2009[en línea]. Ciudad Juárez: SEMAC; 2009 [citado may 2014]. Disponible en: http://www. semac.org.mx/images/stories/libros/Libro\%20SEMAC\%202009.pdf 
38. Instituto de Investigación de Ingenieria de Aragón. Predicción De Carga Postural Por Medio De Un Análisis Biomecánico Y De Esfuerzo Muscular De La Actividad Laboral [en línea]. Zaragoza: ID Ergo; 2011[citado may 2014]. Disponible en: http://www.camarazaragoza.com/prevencion/docs/proyectos/ proyectos3.pdf

39. Coenen $P$, Kingma I, Boot $C$, Faber $G, X u e X$, Bongers $P$, vanDie F. Estimation of low back moments from video analysis: A validation study. J Biomech. 2011:44: 2369-2375.

40. García A, Gadea R, Sevilla M. Ronda E. Validación de un cuestionario para identificar daños y exposición a riesgos ergonómicos en el trabajo. Rev Esp Salud Pública. 2011:85(4): 339-349.

41. Real-Pérez G, García-Dihigo J, Piloto-Fleitas N. El Uso Del Índice De Evaluación Ergonómico Para Evaluar El Trabajo De Las Camareras En La Hotelería. Ing Ind. 2012:33(1): 2-12.

42. Perucha $\mathrm{M}$, Ortega J. Evaluación de las posturas de trabajo como riesgo de carga fisica en el sector Marítimo-Pesquero. INSHT. 2003:(28):11-15.

43. Manero R, Soto L, Rodriguez T. Un modelo simple para la evaluación integral del riesgo a lesiones músculo-esqueléticas (MODSI). MAPFRE MEDICINA. 2005:16(2):12-20.

44. Diniz de Sá $F$, do Nascimento $M$, Carvalho $A$, da Costa J, Adissi P. Comparison Of Methods RULA and REBA For Evaluation Of Postural Stress In Odontological Services. Third International Conference on Production Research Americas' Region 2006 (ICPR-AM06), Curitiba, Jul 30-ago 2, 2006[en línea]. Curitiba: ICPR; 2006[citado may 2014]. Disponible en: http://www. produtronica.pucpr.br/icpram06/Accepted $\% 20$ but $\% 20$ not $\% 20$ coming $\% 20$ for\%20presentation/Paper\%20067.pdf

45. Vigil L, Gutiérrez R, Cáceres W, Collantes H, Beas J. Salud Ocupacional En El Trabajo De Estiba: Los Trabajadores De Mercados Mayoristas De Huancayo. Rev Peru Med Exp Salud Pública. 2007:24(4): 336-342.
46. Gasca M, Rengifo M, Rodríguez E. Evaluación Ergonómica de los Puestos de Trabajo en el Área de Tapas de una Empresa Metalúrgica. Revista Ingenieria Industrial; Actualidad y Nuevas Tendencias. 2008:1(1): 30-42.

47. Rodríguez $E$, Medina $E$, Manero A. Evaluación del nivel de riesgo a lesión músculo-esquelética en el sector automotriz venezolano. Universidad, Ciencia y Tecnología. 2008:12(48): 147-156.

48. Medina $\mathrm{M}$, Hernández J, García J. Ergonomic analysis in public markets in Cortazar Guanajuato. XV Congreso Internacional de Ergonomía SEMAC, Ciudad Juárez, 22-25ene 2009[en línea].México: SEMA; 2009[citado mayo 2014] Disponible en: http://www.semac.org.mx/archivos/congreso11/ EVAL1

49. Fandiño $S$, Peña $C$, Rey $S$, Puentes $D$, Báez L. Condiciones ergonómicas en la práctica de ortodoncia de los residentes de la especialización de ortopedia funcional y ortodoncia de la universidad cooperativa de Colombia, sede Bogotá, durante el primer semestre del 2009. Rev Nac Odontol. 2009:6(10): 49-56.

50. Fernández J, Marley R. Occupational Ergonomics: Emphasis on Identification or Solutions. XV Congreso Internacional de Ergonomía SEMAC, Ciudad Juárez, 22-25ene 2009. México: SEMA; 2009

51. Singh J. Work Posture Assessment In Forging Industry: An Exploratory Study In India. Int J Adv Engine Tech. 2010:1(3): 358-366.

52. García C, Rodríguez E. Evaluación ergonómica en una empresa del sector alimenticio venezolano. Ing Ind. 2010:3(1): 95-108.

53. Vargas $P$, Sánchez $F$, Medina E. Evaluación ergonómica en el área de armado de una empresa cauchera venezolana. Revista Ingeniería Industrial. Actualidad y Nuevas Tendencias. 2010:2(5): 7-22.

54. Batiz $\mathrm{E}, \mathrm{Garcia} \mathrm{L}$, Anzardo 0 . Análise comparativa entre métodos de carregamento de cargas e análise postural de auxiliares de enfermagem. Produção. 2012:22(2): 270-283. 